Article

\title{
Beware of a Fragile Footplate: Lessons from Ossiculoplasty in Patients with Ossicular Anomalies Related to Second Pharyngeal Arch Defects
}

\author{
Sun A Han $『$, Goun Choe, Yoonjoong Kim, Ja-Won Koo®, Byung Yoon Choi and Jae-Jin Song *® \\ Department of Otorhinolaryngology-Head and Neck Surgery, Seoul National University Bundang Hospital, \\ 300 Gumi-dong, Bundang-gu, Seongnam 13620, Korea; hsa226@gmail.com (S.A.H.); \\ delicatewave@gmail.com (G.C.); mdyjkim@gmail.com (Y.K.); jwkoo99@snu.ac.kr (J.-W.K.); \\ choiby@snubh.org (B.Y.C.) \\ * Correspondence: jjsong96@snubh.org or jjsong96@gmail.com; Tel.: +82-31-787-7408; Fax: +82-31-787-4057
}

Received: 20 October 2019; Accepted: 1 December 2019; Published: 3 December 2019

\begin{abstract}
Background and objectives: We review the intraoperative findings and postoperative outcomes of ossiculoplasty in subjects with second pharyngeal arch (SPA)-derived ossicular anomalies. We summarize potential intraoperative complications and recommend precautions that may reduce the risk of fracture. Materials and Methods: Twenty-four patients with SPA-derived ossicular anomalies were included, and pre- and postoperative audiometric results were compared. Results: The mean air conduction threshold $(56.0 \pm 12.4 \mathrm{~dB} H \mathrm{HL})$ was significantly improved 1 month $(27.6 \pm 10.1 \mathrm{~dB} H L)$ and 6 months $(23.8 \pm 13.2 \mathrm{~dB} \mathrm{HL})$ after surgery $(p<0.001)$. The preoperative air-bone gap (ABG) $(40.4 \pm 7.4 \mathrm{~dB}$ HL) was significantly decreased at 1 month (15.1 $\pm 5.9 \mathrm{~dB} \mathrm{HL})$ and 6 months $(11.3 \pm 8.9 \mathrm{~dB} \mathrm{HL})$ postoperation. ABG closure was successful $(<20 \mathrm{~dB} \mathrm{HL})$ in 21 $(87.5 \%)$ patients 6 months after surgery. Intraoperative footplate fractures occurred in 3 of 24 patients. The fractures were managed successfully, and the ABG closure was successful in all cases. Conclusions: The stapes footplate is likely to be relatively thin in subjects with SPA-derived ossicular anomalies because the footplate is partially or totally derived from the SPA. Thus, a fragile footplate should be expected, and care is needed when handling the footplate. However, when complications are overcome, the audiological outcomes are excellent in most cases.
\end{abstract}

Keywords: ossicular anomaly; footplate; ossiculoplasty; second pharyngeal arch

\section{Introduction}

A congenital ossicular anomaly should be suspected when a patient presents with nonprogressive conductive hearing loss with a normal tympanic membrane and no history of infection or trauma. Embryological studies of the development of the middle ear ossicles have shown that the head of the malleus and the body and short process of the incus are derived from the first pharyngeal arch, while the handle of the malleus, the long process of the incus, and the stapes suprastructure (the head and crus) are derived from the second pharyngeal arch [1] (Figure 1A). Early studies showed that the labyrinthine portion (medial half) of the stapes footplate is derived from the mesenchyme of the otic capsule, while the lateral portion is derived from the second pharyngeal arch [1-4] (Figure 1B). However, recent contradictory evidence suggests that the footplate develops independently of the otic capsule, being derived solely from the second pharyngeal arch [5,6] (Figure 1C). 


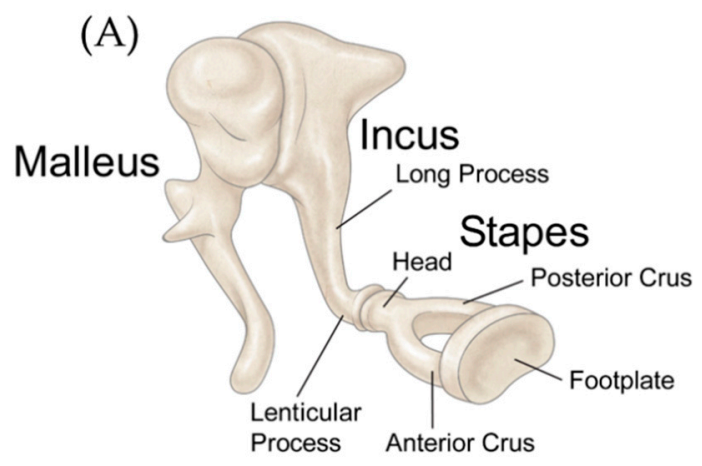

(B)

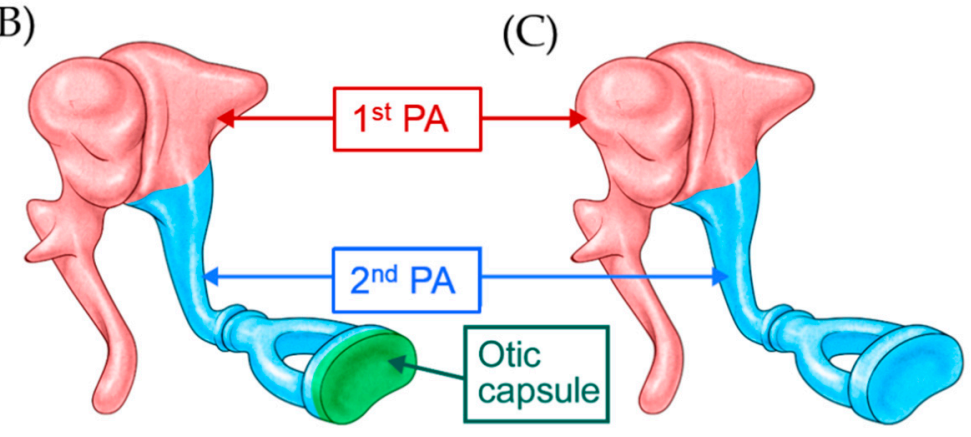

Figure 1. (A) Schematic representation of the ossicular chain; (B) Diagrammatic illustration of the theory suggesting that the medial portion of the footplate is derived from the otic capsule, while the lateral portion is derived from the second pharyngeal arch; (C) Diagrammatic illustration of the theory suggesting that the footplate is derived solely from the second pharyngeal arch. PA: pharyngeal arch.

Several studies concerned with classifying and predicting surgical outcomes in patients with congenital ossicular anomalies have been conducted [7-11]. Although ossiculoplasty is associated with favorable hearing outcomes in patients with congenital ossicular discontinuity and mobile stapes, unexpected intraoperative complications requiring further treatment may occur. In our institution, some patients undergoing ossiculoplasty for a congenital ossicular anomaly experienced intraoperative micro- or gross fracture of the footplate. The common findings in these cases were an absent or hypoplastic incus and a missing stapes suprastructure, indicating that the anomaly was associated with a defect in the second pharyngeal arch.

These unexpected intraoperative events prompted us to perform a retrospective study of patients who had undergone ossiculoplasty for ossicular anomalies associated with the malformation of the second pharyngeal arch. We hypothesized that ossicular anomalies related to the second pharyngeal arch would involve the lateral portion of the footplate, thus necessitating careful treatment. We assessed intraoperative findings and postoperative outcomes in these patients and summarized the potential complications and necessary intraoperative precautions.

\section{Materials and Methods}

\subsection{Study Design}

This study is a retrospective analysis of patients who underwent exploratory tympanotomy and ossiculoplasty for a second-pharyngeal-arch-related ossicular anomaly between April 2015 and December 2018. The study was approved by the Institutional Review Board of the Clinical Research Institute of Seoul National University Bundang Hospital (IRB No. B-XXX), and the requirement for informed consent was waived. A review of the electronic medical records (EMRs), including surgical records, of 108 patients who underwent ossiculoplasty and exploratory tympanotomy during the study period revealed that 24 patients had undergone ossiculoplasty to correct an anomaly attributable to malformation of the second pharyngeal arch (i.e., anomalies of the long process of the incus and/or the 
stapes suprastructure). All of these patients were included in our study. Three experienced surgeons (JWK, BYC, and JJS (Ja-Won Koo, Byung Yoon Choi, and Jae-Jin Song)), each with at least 11 years' experience as an otology specialist, performed all of the ossiculoplasty procedures.

\subsection{Clinical Data Collection}

Clinical data, including demographic characteristics (such as age and sex), the side operated on, and the surgeon who performed the surgery, were obtained from the EMRs. The surgical records were reviewed to identify the types of ossicular anomalies, types of ossiculoplasty performed, and prostheses used. The pure-tone average (PTA) was calculated at $0.5,1,2$, and $3 \mathrm{kHz}$ for air conduction (AC) and bone conduction (BC) [12]. The air-bone gap (ABG) was calculated by subtracting the BC-PTA from the AC-PTA. The patients were followed up 1, 3, 6, and 12 months after surgery, and audiometric tests were performed at each follow-up visit.

All subjects underwent temporal bone computed tomography (ТВCT) using a multidetector $\mathrm{CT}$ scanner (Brilliance iCT; Philips Healthcare, Best, the Netherlands) according to the protocol of our institution [13-18]. The imaging protocol involved helical acquisition with $120 \mathrm{kVp}, 250 \mathrm{mAs}$, and a pitch of 0.825 . Images were obtained at a slice thickness of $0.67 \mathrm{~mm}$ with $0.33 \mathrm{~mm}$ increments and reformatted at a section thickness of $0.7 \mathrm{~mm}$ with no gap. Both axial and coronal reformations were included in the image review.

\subsection{Statistical Analyses}

Statistical tests were performed using SPSS software (version 17.0; SPSS Inc., Chicago, IL, U.S.A.). Paired $t$-tests were used to compare pre- and postoperative hearing thresholds; $p$ values $<0.05$ were deemed to indicate statistical significance in all analyses.

\section{Results}

\subsection{Demographic Characteristics and TBCT Findings}

The study included 24 patients with ossicular anomalies due to defective development of the second pharyngeal arch. Of the patients, $13(54 \%)$ were male, and $11(46 \%)$ were female. The average age at surgery was $31.5 \pm 15.1$ years. In total, $11(46 \%)$ surgeries were performed on the right ear and $13(54 \%)$ on the left ear. Of the 24 surgeries, $13(54 \%)$ were performed by JJS, $6(25 \%)$ by JWK, and 5 $(21 \%)$ by BYC. The mean follow-up duration was $10.5 \pm 8.9$ months.

On axial and coronal scans of TBCT, all subjects were found to have suspicious congenital ossicular anomalies involving the long process of the incus and the suprastructure of the stapes. However, due to the limited resolution of TBCT, we could not evaluate the risk of footplate fracture preoperatively.

\subsection{Postoperative Hearing Outcomes}

The preoperative AC-PTA was $56.0 \pm 12.4$ decibels hearing level (dB HL), and the BC-PTA and ABG were $15.7 \pm 9.6$ and $40.4 \pm 7.4 \mathrm{~dB}$ HL, respectively. At the 1-month postoperative follow-up, the mean AC-PTA $(27.6 \pm 10.1 \mathrm{~dB}$ HL) was significantly lower than the preoperative value $(p<0.001$; Figure 2A), the BC-PTA was $12.4 \pm 9.4 \mathrm{~dB} \mathrm{HL}$, and the ABG (15.1 $\pm 5.9 \mathrm{~dB} \mathrm{HL})$ was significantly less than the preoperative value $(p<0.001$; Figure $2 B, C)$. Furthermore, significant improvements were observed in the AC-PTA, BC-PTA, and ABG 6 months after surgery $(23.8 \pm 13.2,12.5 \pm 9.4$, and $11.3 \pm 8.9 \mathrm{~dB}$ HL, respectively; $p<0.05$; Figure 2). In total, 21 (87.5\%) patients had achieved successful ABG closure ( $<20 \mathrm{~dB}$ HL) [19] at 6 months after surgery. Subjects with congenital ossicular anomalies who have undergone ossiculoplasty are routinely followed up until 12 months postoperation at our institution, but only 11 of 24 patients $(45.8 \%)$ in the current case series were followed up until 12 months postoperation, probably due to subjective satisfaction and no further need to revisit the outpatients' clinic after 6 months postoperation. The mean 12-month postoperative AC-PTA ( $26.6 \pm 15.1 \mathrm{~dB}$ HL) and $\mathrm{ABG}(13.2 \pm 9.8 \mathrm{~dB} \mathrm{HL})$ of these 11 subjects showed significant improvement as compared with 
the preoperative AC-PTA $(57.6 \pm 14.1 \mathrm{~dB}$ HL) and ABG $(40.7 \pm 8.2 \mathrm{~dB}$ HL) $(p<0.01)$. Meanwhile, the 12-month postoperative BC-PTA $(15.7 \pm 9.6 \mathrm{~dB} \mathrm{HL})$ of these 11 subjects showed a tendency of improvement as compared with the preoperative BC-PTA $(16.8 \pm 11.6 \mathrm{~dB} \mathrm{HL})$, but this difference failed to reach statistical significance $(p=0.089)$. The most commonly performed ossiculoplasty was total ossicular replacement prosthesis (TORP; $n=19$ ), followed by partial ossicular replacement prosthesis (PORP), incus-footplate assembly (IFA), and malleostapedotomy. Although the stapes suprastructure was rudimentary in the two patients who underwent PORP reconstruction, it was sufficient to enable placement of the prosthesis.

(A)

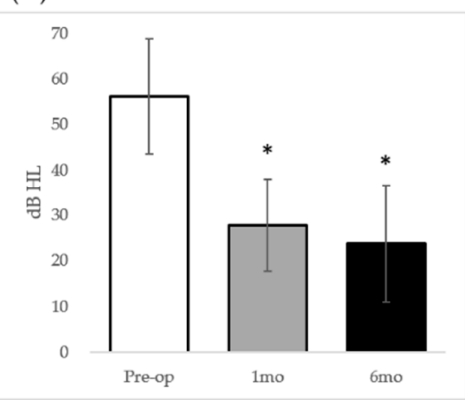

(B)

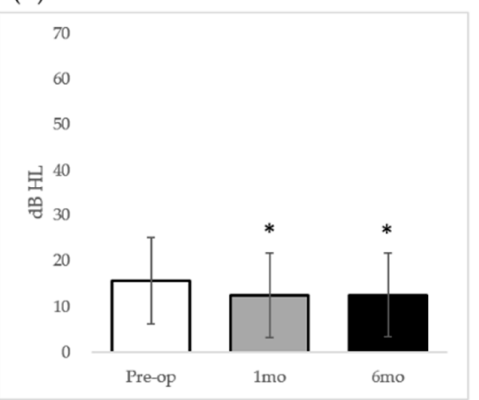

(C)

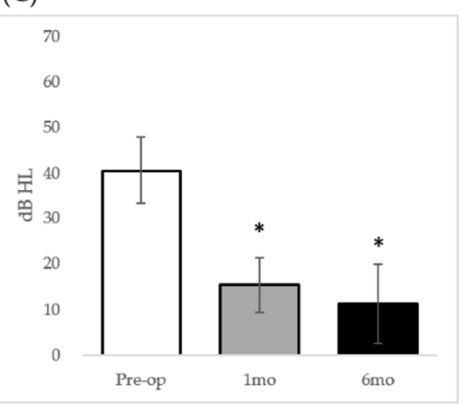

Figure 2. Changes in audiometric parameters after surgery. (A) Air conduction (AC) pure-tone average (PTA); (B) Bone conduction (BC) PTA; (C) Air-bone (AB) gap. * $p$ values less than 0.05; Pre-op: preoperative average; $1 \mathrm{mo}$ : 1-month postoperative average; $6 \mathrm{mo}$ : 6-month postoperative average.

\subsection{Intraoperative Complications}

Intraoperative complications occurred in three cases, although no patients experienced postoperative complications. All three cases of intraoperative complications involved fracture of the stapes footplate. The first case was a 42-year-old male who was missing the long process of the incus and stapes suprastructure. The footplate sustained a microfracture while its mobility was being checked, despite being manipulated in the conventional manner using gentle pressure. The fractured portion of the footplate was covered with tragal perichondrium and soft tissue, and a TORP was then placed on the reconstructed footplate. No change was observed in the postoperative BC thresholds, and the ABG improved from 40 to $12.5 \mathrm{~dB}$ HL (Figure 3A). The second case was a 35-year-old male with conductive hearing loss and a preoperative $\mathrm{ABG}$ of $47.5 \mathrm{~dB}$ HL. A malformed incus and a floating stapes suprastructure were identified intraoperatively. After the floating stapes suprastructure was removed, gentle pressure applied to the footplate to check mobility caused a fracture in the posterior portion. Because the defect was large and the long process of the incus was missing, a malleostapedotomy using a piston-wire prosthesis (length $5.8 \mathrm{~mm}$; diameter $0.4 \mathrm{~mm}$ ) was performed. No postoperative complications were observed in this case. The $B C$ threshold remained the same, while the ABG improved from $47.5 \mathrm{~dB}$ HL preoperatively to $17.5 \mathrm{~dB}$ HL postoperatively (Figure 3B). The third case was a 61-year-old female with hypoplastic incus and stapes footplate and with no suprastructure identified intraoperatively. The footplate was fractured while checking its mobility. The damaged footplate was sealed using soft tissue and fibrin glue. After reconstruction of the footplate, a TORP (length $4.75 \mathrm{~mm}$ ) was implanted. The ABG was reduced from 47.5 to $12.5 \mathrm{~dB} \mathrm{HL}$, with no change in the BC threshold (Figure 3C). 
(A)

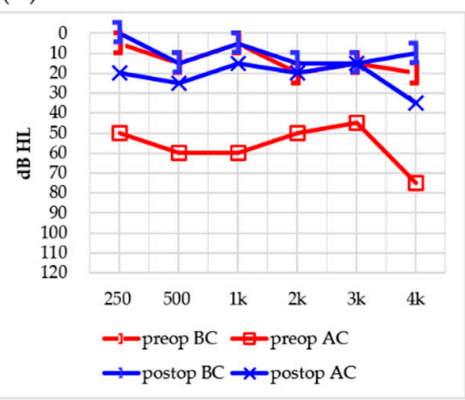

(B)

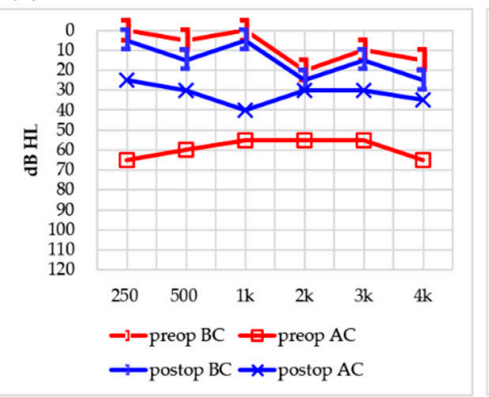

(C)

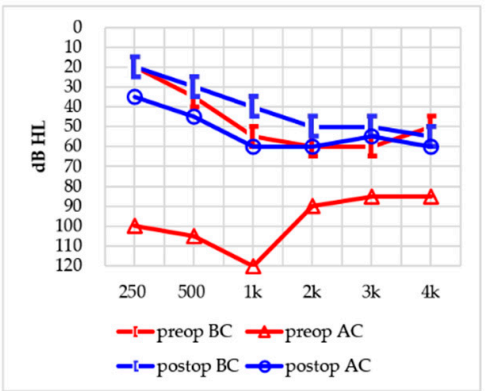

Figure 3. Comparison of preoperative (preop) and postoperative (postop) audiometric outcomes in patients with intraoperative complications. (A) Case 1, a 42-year-old male; (B) Case 2, a 35-year-old male; (C) Case 3, a 61-year-old female. BC: bone conduction; AC: air conduction.

\section{Discussion}

We reviewed the intraoperative findings and postoperative outcomes of ossiculoplasty in patients with ossicular anomalies attributable to abnormal development of the second pharyngeal arch. Furthermore, we described three cases of intraoperative iatrogenic footplate fracture and the postoperative outcomes.

\subsection{Postoperative Audiometric Outcomes in Subjects with Second Pharyngeal Arch Related Ossicular Anomalies}

Congenital ossicular anomaly is a rare cause of conductive hearing loss, with a reported incidence of less than 1 per 15,000 births [20]. In 1993, Teunissen and Cremers classified congenital ossicular anomalies into four categories, based on surgical findings in 144 ears with middle ear malformations: congenital isolated stapes ankylosis (Class 1), congenital stapes ankylosis in combination with a congenital anomaly of the ossicular chain (Class 2), a congenital anomaly of the ossicular chain accompanied by at least a mobile stapes footplate (Class 3), and aplasia or severe dysplasia of the oval window or round window (Class 4) [1]. This classification system was further refined by Park et al. [10] according to the presence or absence of the stapes suprastructure. A previous study found that the most common ossicular anomaly was a mobile stapes with a defective long process of the incus and a missing suprastructure, corresponding to Class 3 of the Teunissen and Cremers classification system [21]. Thus, it follows that ossicular anomalies resulting from malformation of the second pharyngeal arch are the most common anomalies because the long process of the incus and stapes are derived from the second pharyngeal arch.

Several studies reported achieving significant hearing gains by exploratory tympanotomy and ossiculoplasty in subjects with congenital ossicular anomalies $[11,19,22,23]$. The rate of successful ABG closure $(<20 \mathrm{~dB}$ HL) achieved at 6 months postoperation in our study following exploratory tympanotomy and ossiculoplasty, i.e., $87.5 \%$, is comparable to that of previous studies, which reported closure rates ranging from $56 \%$ to $70 \%[11,19,22,23]$. Although four types of surgery were performed in our cohort (TORP, PORP, IFA, and malleostapedotomy), the small sample size in each group (except for the TORP group) prevented us from determining whether one type was superior to the others.

\subsection{Risk Factors Associated with Ossiculoplasty in Subjects with Second Pharyngeal Arch Related Ossicular Anomalies}

Of the 24 cases in our study, $3(12.5 \%)$ sustained iatrogenic footplate fractures while the mobility of the footplate was being checked, requiring reconstruction. Although these cases were managed successfully, and the postoperative audiological outcomes were satisfactory, we investigated the possible reasons for frequent fractures of the footplate and the intraoperative precautions that should be taken to reduce the risk thereof. 
The developmental origin of the stapes footplate is controversial. While previous investigators have argued that the labyrinthine portion of the footplate is derived from the otic capsule, while the lateral portion is derived from the second pharyngeal arch [1-4], recent findings suggest that the entire footplate is derived from the second pharyngeal arch $[5,6]$. Nevertheless, given that the stapes footplate is at least partially derived from the second pharyngeal arch, it follows that patients with ossicular anomalies related to a defect in the second pharyngeal arch who are missing the long process of the incus and stapes suprastructure are likely to have an abnormally thin footplate (Figure 4A,B). Thus, when correcting ossicular anomalies caused by malformation of the second pharyngeal arch, surgeons should expect the footplate to be fragile and refrain from exerting excessive force when checking its mobility or implanting an ossicular prosthesis. Furthermore, surgeons should be prepared to perform malleostapedotomy or stapes reconstruction and TORP with ossiculoplasty in these patients.

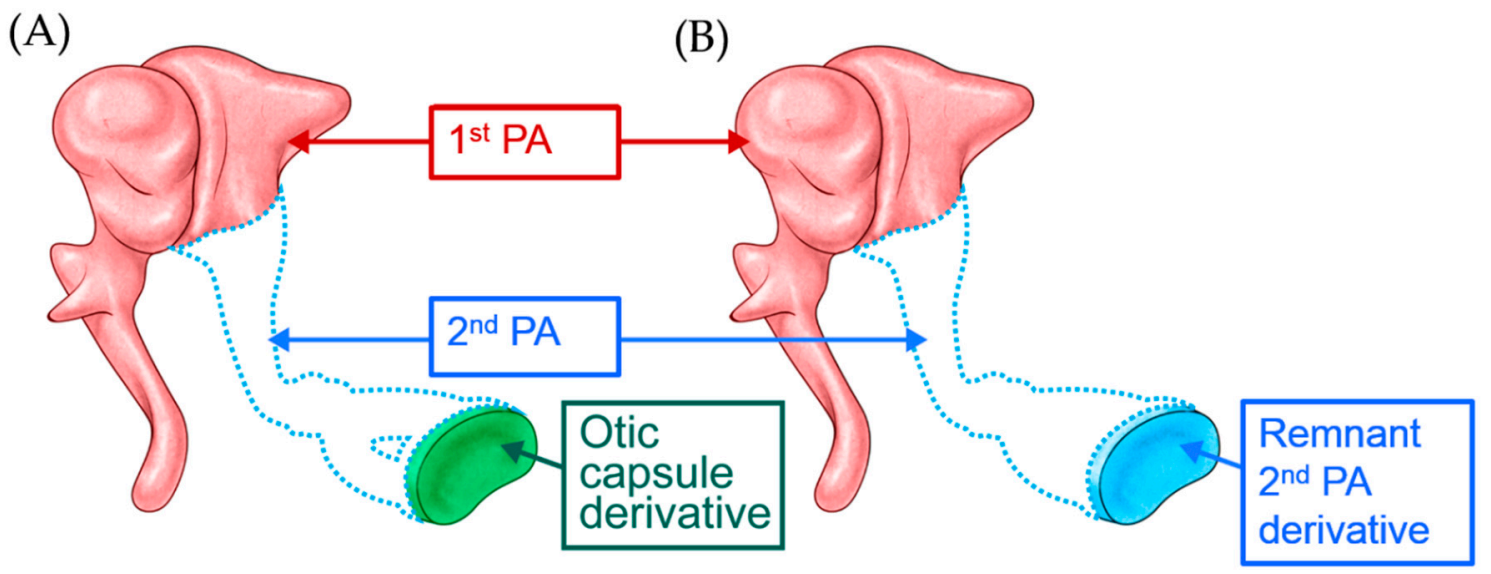

Figure 4. The stapes footplate is (A) partially or (B) totally derived from the second PA.

\subsection{Study Limitations and Future Directions}

To our knowledge, this study is the first to report unique intraoperative complications of ossiculoplasty in patients with ossicular anomalies due to malformation of the second pharyngeal arch. However, our study had several limitations. First, although we have proposed several possible reasons for frequent footplate fracture, none have been histologically or radiologically confirmed, given the limited resolution of TBCT. Further assessment of footplate thickness using postmortem histological analyses or the high-spatial-resolution TBCT introduced in a recent cadaveric study [24] is needed to support our findings. Second, we can only speculate on the possible causes of a thin footplate, given that no consensus has been reached regarding the developmental origin of the stapes. It may be that future embryological studies confirming the origin of the ossicles will support our findings. Moreover, histological or embryological comparisons of cases with missing or mobile footplates versus those with no long process of the incus or stapes suprastructure may further our understanding of congenital ossicular anomalies.

\section{Conclusions}

Our findings suggest that patients with ossicular anomalies attributable to malformation of the second pharyngeal arch are likely to present with a relatively thin footplate. Thus, a fragile footplate should be expected, and care must be taken to avoid exerting excessive force on the footplate when checking mobility or placing an ossicular prosthesis on the footplate. However, when these complications are overcome, the audiological outcomes after exploratory tympanotomy and ossiculoplasty are excellent in most cases. Furthermore, with proper management, patients with an intraoperative footplate fracture can achieve successful ABG closure. 
Author Contributions: Conceptualization, J.-J.S., S.A.H.; formal analysis, G.C., Y.K.; writing—original draft preparation, S.A.H., J.-J.S.; writing-reviewing and editing, J.-W.K., B.Y.C.

Funding: This work was supported by a grant from the National Research Foundation of Korea (NRF) grant funded by the Korea government (MSIP) (grant number NRF-2019R1A2C2004941).

Conflicts of Interest: The authors declare no conflict of interest.

\section{References}

1. Altmann, F. Problem of so-called congenital atresia of the ear; histologic report of a new case. Arch. Otolaryngol. 1949, 50, 759-788. [CrossRef] [PubMed]

2. Anson, B.J.; Cauldwell, E.W. Stapes, fistula ante fenestram and associated structures in man; from the fetus of $160 \mathrm{~mm}$. (5 months) to newborn infant. Arch. Otolaryngol. 1948, 48, 263-300. [CrossRef] [PubMed]

3. Anson, B.J.; Hanson, J.S.; Richany, S.F. Early embryology of the auditory ossicles and associated structures in relation to certain anomalies observed clinically. Ann. Otol. Rhinol. Laryngol. 1960, 69, 427-447. [CrossRef] [PubMed]

4. Richany, S.F.; Bast, T.H.; Anson, B.J. The development and adult structure of the malleus, incus and stapes. Ann. Otol. Rhinol. Laryngol. 1954, 63, 394-434. [CrossRef]

5. Rodriguez-Vazquez, J.F. Development of the stapes and associated structures in human embryos. J. Anat. 2005, 207, 165-173. [CrossRef]

6. Louryan, S.; Vanmuylder, N.; Resimont, S. Ectopic stapes: A case report with embryologic correlations. Surg. Radiol. Anat. 2003, 25, 342-344. [CrossRef]

7. Funasaka, S. Congenital ossicular anomalies without malformations of the external ear. Arch. Otorhinolaryngol. 1979, 224, 231-240. [CrossRef]

8. Hanson, J.R.; Anson, B.J.; Strickland, E.M. Branchial sources of the auditory ossicles in man. II. Observations of embryonic stages from $7 \mathrm{~mm}$. to $28 \mathrm{~mm}$. (CR length). Arch. Otolaryngol. 1962, 76, 200-215. [CrossRef]

9. Henner, R.; Buckingham, R.A. The recognition and surgical treatment of congenital ossicular defects. Laryngoscope 1956, 66, 526-539. [CrossRef]

10. Park, K.; Choung, Y.H. Isolated congenital ossicular anomalies. Acta Otolaryngol. 2009, 129, 419-422. [CrossRef]

11. Teunissen, E.B.; Cremers, W.R. Classification of congenital middle ear anomalies. Report on 144 ears. Ann. Otol. Rhinol. Laryngol. 1993, 102, 606-612. [CrossRef] [PubMed]

12. American Academy of Otolaryngology-Head and Neck Surgery Ffoundation, Inc. Committee on Hearing and Equilibrium guidelines for the evaluation of results of treatment of conductive hearing loss. Otolaryngol.-Head Neck Surg. 1995, 113, 186-187. [CrossRef]

13. Shim, Y.J.; Bae, Y.J.; An, G.S.; Lee, K.; Kim, Y.; Lee, S.Y.; Choi, B.Y.; Choi, B.S.; Kim, J.H.; Koo, J.W.; et al. Involvement of the Internal Auditory Canal in Subjects With Cochlear Otosclerosis: A Less Acknowledged Third Window That Affects Surgical Outcome. Otol. Neurotol. 2019, 40, e186-e190. [CrossRef] [PubMed]

14. Lee, S.Y.; Song, S.K.; Park, S.J.; Park, H.G.; Choi, B.Y.; Koo, J.W.; Song, J.J. Jugular Bulb Resurfacing With Bone Cement for Patients With High Dehiscent Jugular Bulb and Ipsilateral Pulsatile Tinnitus. Otol. Neurotol. 2019, 40, 192-199. [CrossRef] [PubMed]

15. Bae, Y.J.; Shim, Y.J.; Choi, B.S.; Kim, J.H.; Koo, J.W.; Song, J.J. “Third Window” and "Single Window” Effects Impede Surgical Success: Analysis of Retrofenestral Otosclerosis Involving the Internal Auditory Canal or Round Window. J. Clin. Med. 2019, 8, 1182. [CrossRef] [PubMed]

16. Song, J.J.; Hong, S.K.; Lee, S.Y.; Park, S.J.; Kang, S.I.; An, Y.H.; Jang, J.H.; Kim, J.S.; Koo, J.W. Vestibular Manifestations in Subjects With Enlarged Vestibular Aqueduct. Otol. Neurotol. 2018, 39, e461-e467. [CrossRef]

17. Bae, Y.J.; Song, J.J.; Choi, B.S.; Kang, Y.; Kim, J.H.; Koo, J.W. Differentiation Between Intralabyrinthine Schwannoma and Contrast-enhancing Labyrinthitis on MRI: Quantitative Analysis of Signal Intensity Characteristics. Otol. Neurotol. 2018, 39, 1045-1052. [CrossRef]

18. Sunwoo, W.; Jeon, Y.J.; Bae, Y.J.; Jang, J.H.; Koo, J.W.; Song, J.J. Typewriter tinnitus revisited: The typical symptoms and the initial response to carbamazepine are the most reliable diagnostic clues. Sci. Rep. 2017, 7, 10615. [CrossRef]

19. Quesnel, S.; Benchaa, T.; Bernard, S.; Martine, F.; Viala, P.; Van Den Abbeele, T.; Teissier, N. Congenital middle ear anomalies: Anatomical and functional results of surgery. Audiol. Neurootol. 2015, 20, 237-242. [CrossRef] 
20. Farrior, J.B. Surgical management of congenital conductive deafness. South. Med. J. 1987, 80, 450-453. [CrossRef]

21. Cousins, V.C.; Milton, C.M. Congenital ossicular abnormalities: A review of 68 cases. Am. J. Otol. 1988, 9, 76-80. [PubMed]

22. Sakamoto, T.; Kakigi, A.; Kashio, A.; Kanaya, K.; Suzuki, M.; Yamasoba, T. Evaluation of the Carhart effect in congenital middle ear malformation with both an intact external ear canal and a mobile stapes footplate. ORL J. Otorhinolaryngol. Relat Spec. 2011, 73, 61-67. [CrossRef] [PubMed]

23. Thomeer, H.G.; Kunst, H.P.; Cremers, C.W. Congenital ossicular chain anomalies associated with a mobile stapes footplate: Surgical results for 23 ears. Ann. Otol. Rhinol. Laryngol. 2012, 121, 275-281. [CrossRef]

24. Zhou, W.; Lane, J.I.; Carlson, M.L.; Bruesewitz, M.R.; Witte, R.J.; Koeller, K.K.; Eckel, L.J.; Carter, R.E.; McCollough, C.H.; Leng, S. Comparison of a Photon-Counting-Detector CT with an Energy-Integrating-Detector CT for Temporal Bone Imaging: A Cadaveric Study. AJNR Am. J. Neuroradiol. 2018, 39, 1733-1738. [CrossRef]

(C) 2019 by the authors. Licensee MDPI, Basel, Switzerland. This article is an open access article distributed under the terms and conditions of the Creative Commons Attribution (CC BY) license (http://creativecommons.org/licenses/by/4.0/). 